\title{
609.
}

\section{ON THE ANALYTICAL FORMS CALLED FACTIONS.}

[From the Report of the British Association for the Advancement of Science, (1875), p. 10.]

A FACTION is a product of differences such that each letter occurs the same number of times; thus we have a quadrifaction where each letter occurs twice, a cubifaction where each letter occurs three times, and so on. A broken faction is one which is a product of factions having no common letter; thus

$$
(a-b)^{2}(c-d)(d-e)(e-c)
$$

is a broken quadrifaction, the product of the quadrifactions

$$
(a-b)^{2} \text { and }(c-d)(d-e)(e-c) .
$$

We have, in regard to quadrifactions, the theorem that every quadrifaction is a sum of broken quadrifactions such that each component quadrifaction contains two or else three letters. Thus we have the identity

$$
2(a-b)(b-c)(c-d)(d-a)=(b-c)^{2} \cdot(a-d)^{2}-(c-a)^{2} \cdot(b-d)^{2}+(a-b)^{2} \cdot(c-d)^{2},
$$

which verifies the theorem in the case of a quadrifaction of four letters; but the verification even in the next following case of a quadrifaction of five letters is a matter of some difficulty.

The theory is connected with that of the invariants of a system of binary quantics. 